\title{
Aspectos epidemiológicos da tuberculose na região norte de saúde do estado do Espírito Santo (2015 - 2019)
}

\author{
Epidemiological aspects of tuberculosis in the northern health region of the state of espirito santo (2015 - 2019) \\ Aspectos epidemiológicos de la tuberculosis en la región sanitaria norte del estado de espírito santo (2015-2019)
}

\section{RESUMO}

Objetivo: Descrever aspectos epidemiológicos da tuberculose na região norte de saúde do estado do Espírito Santo no intervalo de 2015 a 2019. Método: Trata-se de um estudo epidemiológico ecológico, com componente descritivo e analítico, do tipo série histórica. Para análise dos dados, foram calculados coeficientes de incidência (100.000 habitantes) e tendência quadrática para região norte. Resultados: durante o período avaliado, na Região Norte foram notificados 559 casos de tuberculose (2015-2019), onde foi possivel observar um declínio do agravo nos anos de 2015 à 2017 voltando a crescer pós esse período. 0 coeficiente de

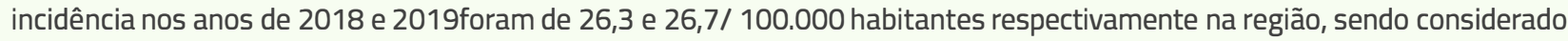
abaixo da média nacional para mesmo período. Conclusão: A região apresenta bom desempenho na redução dos índices de incidência para o agravo, entretanto, é possível observar necessidades de modelação de políticas de saúde voltadas para o agravo. DESCRITORES: Tuberculose; Promoção da Saúde; Saúde Pública; Política de Saúde

\section{ABSTRACT}

Objective: To describe epidemiological aspects of tuberculosis in the northern health region of the state of Espírito Santo between 2015 and 2019. Method: This is an ecological epidemiological study, with a descriptive and analytical component, of the historical series type. For data analysis, incidence coefficients $(100,000$ inhabitants) and quadratic trend for the northern region were calculated. Results: during the period evaluated, in the North Region, 559 cases of tuberculosis were reported (20152019), where it was possible to observe a decline in the disease in the years 2015 to 2017, growing again after this period. 2018 and 2019 were 26.3 and $26.7 / 100,000$ inhabitants respectively in the region, being considered below the national average for the same period. Conclusion: The region has a good performance in reducing incidence rates for the disease, however, it is possible to observe the needs for modeling health policies aimed at the disease.

DESCRIPTORS: Tuberculosis; Health Promotion; Public Health; Health Policy.

\section{RESUMEN}

Objetivo: Describir aspectos epidemiológicos de la tuberculosis en la región sanitaria norte del estado de Espírito Santo entre 2015 y 2019. Método: Se trata de un estudio epidemiológico ecológico, con componente descriptivo y analítico, del tipo serie histórica. Para el análisis de los datos se calcularon coeficientes de incidencia ( 100.000 habitantes) y tendencia cuadrática para la región norte. Resultados: durante el período evaluado, en la Región Norte se notificaron 559 casos de tuberculosis (20152019), donde se pudo observar una disminución de la enfermedad en los años 2015 a 2017, creciendo nuevamente luego de este período. 2018 y 2019 fueron 26,3 y 26,7 / 100.000 habitantes respectivamente en la región, considerándose por debajo del promedio nacional para el mismo período. Conclusión: La región tiene un buen desempeño en la reducción de las tasas de incidencia de la enfermedad, sin embargo, es posible observar las necesidades de modelar políticas de salud dirigidas a la enfermedad.

DESCRIPTORES: Tuberculosis; Promoción de la Salud; Salud Pública; Política de salud.

RECEBIDO EM: 28/07/2021 APROVADO EM: 11/08/2021 


\section{artigo}

Sotero, R. L., Gouvea, R. R., Cardoso, M. L., Gomes, A. M., Forza, D. H.,Santos, G. L.

Aspectos epidemiológicos da tuberculose na região norte de saúde do estado do Espírito Santo (2015 - 2019)

\section{RAFAELA LIRIO SOTERO}

Enfermeira. Especialista em enfermagem do trabalho e saúde ocupacional. Residente do programa de residência multiprofissional em saúde coletiva com ênfase em vigilância em saúde do Instituto Capixaba de Ensino Pesquisa e Inovação em Saúde da Secretaria Estadual de Saúde do Espírito Santo. Mestranda em Gestão Pública pela Universidade Federal do Espírito Santo. ORCID: 0000-0001-8127-6129

\section{RAFAEL RUY GOUVEA}

Biólogo. Residente do programa de residência multiprofissional em saúde coletiva com ênfase em vigilância em saúde do Instituto Capixaba de Ensino Pesquisa e Inovação em Saúde da Secretaria Estadual de Saúde do Espírito Santo.

ORCID: 0000-0002-7950-9453

\section{MAYARA LIMA CARDOSO}

Médica Veterinária. Residente do programa de residência multiprofissional em saúde coletiva com ênfase em vigilância em saúde do Instituto Capixaba de Ensino Pesquisa e Inovação em Saúde da Secretaria Estadual de Saúde do Espírito Santo.

ORCID: 0000-0002-5753-265

\section{ALESSANDRO MENDES GOMES}

Médico Veterinário. Especialista em Gestão em Serviços de Saúde pela UNIVEN. Especialista em Medicina Veterinária do Coletivo - Prominas. Referência Técnica da Vigilância Ambiental da Superintendência Regional de Saúde Norte do estado do Espírito Santo.

ORCID:0000-0001-5294-8547.

\section{DALZA HELENA FORZA}

Bióloga. Pós Graduada em Gestão da Saúde Pública pela Faculdade de Nanuque MG - FANAN. Especialista em Vigilância Sanitária e Epidemiológica pela Universidade de Ribeirão Preto - SP - UNAERP. Referência técnica do agravo de tuberculose da Superintendência Regional de Saúde Norte do estado do Espírito Santo.

ORCID: 0000-0003-1559-078X

\section{GABRIELA LIMA SANTOS}

Médica Veterinária. Residente do programa de residência multiprofissional em saúde coletiva com ênfase em vigilância em saúde do Instituto Capixaba de Ensino Pesquisa e Inovação em Saúde da Secretaria Estadual de Saúde do Espírito Santo.

ORCID: 0000-0003-4618-6966

\section{INTRODUÇÃO}

Doença de etiologia infectocontagiosa a tuberculose (TB) é causada pelo Mycobacterium tuberculosis e apesar de se tratar de um agravo milenar, essa, ainda apresenta grande importância no cenário sanitário a nível mundial por se tratar de uma doença negligenciada. Dados da Organização Mundial da Saúde (OMS) sugerem que tuberculose é a principal causa de morte por um único agente infeccioso em todo o mundo14. Estima-se que no ano de 2019, em todo mundo, cerca de dez milhões de pessoas desenvolveram TB. Para o ano de 2020, o Brasil registrou 66.819 mil casos da doença, apresentando um coeficiente de incidência de 3,16/100.000 mil habitantes12.Dessa forma, o Brasil continua entre os 30 países que apresentam uma alta carga
Dados da

Organização

Mundial da Saúde

(OMS) sugerem

que tuberculose é a

principal causa de

morte por um único

agente infeccioso

em todo o mundo para o agravo e coinfecção TB-HIV, sendo considerado prioritário para o controle da doença no mundo pela $\mathrm{OMS}^{13}$.

Ainda no ano de 2017 o Ministério da Saúde (MS) do Brasil aderiu à meta da OMS intitulada "Estratégia pelo Fim da Tuberculose", que sugere que para que haja uma redução da incidência da TB para menos de 10 casos por $100 \mathrm{mil} \mathrm{hab.} \mathrm{até} 2035$, seria necessária uma redução global de 4 a $5 \%$ ao ano (SVS; MS, 2019). No entanto, estudos comprovam que no ano de 2018 havia um número estimado de 10 milhões de novos casos de tuberculose em todo o mundo, e 1,5 milhōes de pessoas morreram devido à doença, onde podemos observar um aumento significativo da estimativa de mortalidade pela doença em relação ao ano anterior como mencionado. No mesmo ano no Brasil, a incidência de tuberculose 
foi de 45 casos/100.000 habitantes, e a taxa de mortalidade relacionada à tuberculose foram de 2,3 óbitos/100.000 habitantes, longe do que é preconizado pela OMS para que haja redução do agravo ${ }^{2}$.

Em consonância com a Estratégia pelo Fim da Tuberculose, em 2017, o MS, por meio da Coordenação Geral do Programa Nacional de Controle da Tuberculose (CGPNCT), lançou então o Plano Nacional pelo Fim da Tuberculose como Problema de Saúde Pública (Plano Nacional), no qual foi realizado um remodelamento geográfico para que a distribuição dos municípios brasileiros fossem divididos em oito cenários a partir da similaridade dos contextos socioeconômicos e capacidade de implementação das ações de controle da TB associados à incidência da doença ${ }^{4}$.

O estado do Espírito Santo aderiu e adotou medidas para enfrentamento da doença por meio do programa estadual de controle da tuberculose, que dentre as diversas competências possuem as de consolidar e analisar os dados gerados pelo sistema de informação, oferecendo informações por meio de boletins ou informes, além de utilizá-las para fins de planejamento, monitoramento e avaliação. Realizar avaliação operacional e epidemiológica das ações do programa em âmbito estadual. Promover e participar da capacitação de recursos humanos na área de tuberculose, fomentando a integração de instituições de ensino e serviço ${ }^{4}$. Assessorar as coordenadorias regionais na implantação e/ou implementação do Programa de Controle da Tuberculose nos municípios. Manter estreita articulação com o Laboratório de Referência Estadual e Regional, participar do planejamento das ações de diagnostico bacteriológico e controle de qualidade. Manter estreita interação com a esfera técnico-operacional, especialmente com as unidades de referência secundárias e terciárias ${ }^{4}$.

No estado todo no ano de 2019 foram notificados 1129 casos da doença e 73 casos confirmados para óbito em decorrência da tuberculose, apresentando um coeficiente de mortalidade de 1,8/100 mil hab. ${ }^{5}$. Na região norte de saúde segundo o Plano Diretor de Regionalização de 2011 no ano de
2019 foram observados 115 casos notificados para tuberculose, o que corresponde a $21 \%$ do panorama estadual, e um quantitativo de três óbitos em decorrência da doença5. O objetivo consiste em descrever aspectos epidemiológicos da tuberculose na região norte de saúde do estado do Espírito Santo nos últimos cinco anos que antecede a pesquisa: $2015-2019$.

Diante do exposto justifica-se a relevância desse estudo uma vez que conhecer o cenário clínico e epidemiológico da distribuição de casos da tuberculose enquanto importante problema de saúde pública e coletiva viabiliza por parte da gestão a criação de medidas mitigadoras para intervenções centradas na real necessidade de saúde da região no formato usuário-centrado, e consequentemente mudanças positivas na atual situação sanitária.

\section{MÉTODOS}

Trata-se de um estudo epidemiológico ecológico, com componente descritivo e analítico, do tipo série histórica, em que os casos incluídos no estudo foram descritos $\mathrm{e}$ analisados de forma agregada por ano e período de estudo.

O local do estudo consiste no território definido por região norte de saúde conforme um PDR (Plano diretor de Regionalização) publicado em 2011 pelo estado do Espírito Santo. O PDR divide o estado em regiões de saúde, sendo a região norte de saúde composta por 14 municípios, sendo eles: Água Doce do Norte, Barra de São Francisco, Boa Esperança, Conceição da Barra, Ecoporanga, Jaguaré, Montanha, Mucurici, Nova Venécia, Pedro Canário, Pinheiros, Ponto Belo, São Mateus e Vila Pavão4.

O estudo foi realizado nos meses de agosto de 2020 a junho do ano de 2021, a considerar o período para a coleta de dados $1^{\circ}$ de janeiro de 2015 a 31 de dezembro de 2019. Para a coleta de dados foram utilizados os casos notificados no Sistema de Informação de Agravos de Notificação (SINAN) através do sistema de tabulação TABNET do banco de dados do Sistema Único de Saúde (SUS) o DATASUS. As definições de caso de TB usadas foram às recomendadas pelo Ministério da Saúde (MS):

Critério laboratorial: todo caso que, independentemente da forma clínica, apresenta pelo menos uma amostra positiva de baciloscopia, de cultura ou de teste rápido molecular para TB (TRM-TB) ${ }^{5}$;

Critério clínico-epidemiológico: todo caso que não preenche o critério de confirmação laboratorial acima descrito, mas que recebeu o diagnóstico de TB ativa, levando em consideração dados clínico-epidemiológicos ${ }^{5}$.

Foram definidos ainda critérios de inclusão e critérios de exclusão para seleção dos dados da pesquisa, sendo critérios de inclusão: qualquer indivíduo com a variável de diagnóstico confirmada para tuberculose que se enquadre nos critérios utilizados pelo MS e que tenha a variável local de residência situada em qualquer município que corresponde à região norte de saúde do estado do Espírito Santo5.

Para os cálculos de incidência, foram utilizadas as bases populacionais do Instituto

Brasileiro de Geografia e Estatística (IBGE) e dados da Superintendência Regional de Saúde de São Mateus onde foi utilizado o número de notificações disponíveis dividido pela população daquele ano em específico x 100.000 habitantes.

Alcançado os resultados preliminares, os dados coletados foram inseridos, organizados e analisados em planilhas de Excel (Windows 10 - Home Single Language). Os coeficientes de incidência foram analisados em um período de cinco anos da série histórica (tendência temporal). Além disso foi produzido o cálculo da tendência quadrática, modelo proposto para ajustar a análise das séries temporais.

Onde,

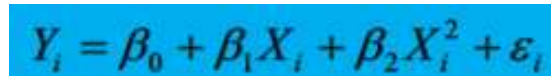

O modelo de tendência quadrática foi calculado em cima do coeficiente de incidência de cada ano com o X codificado (ano), utilizando o software Excel para tabulação, cálculo e juntamente com o gráfico 


\section{artigo}

Sotero, R. L., Gouvea, R. R., Cardoso, M. L., Gomes, A. M., Forza, D. H., Santos, G. L.

Aspectos epidemiológicos da tuberculose na região norte de saúde do estado do Espírito Santo (2015 - 2019)

de dispersão adequado para o modelo de estudo para análise de tendências quadráticas.

A produção de gráficos de notificação $\mathrm{e}$ coeficiente de incidência foi realizada com o auxílio do software estatístico SIGMA PLOT, Scientific Data Analysis and Graphing Software. Versão 12.0. Systat Software Inc, 2013. O cálculo do coeficiente de incidência foi realizado com os dados obtidos no SINAN (TabWin). Trata-se de uma pesquisa com levantamento de dados em bases secundárias que não inferem diretamente na ética humana, portanto conforme a resolução 466/12 que aprova as "diretrizes e normas regulamentadoras de pesquisas envolvendo seres humanos".

\section{RESULTADOS}

Em 2020 o Estado do Espírito Santo apresentou $1176 \mathrm{mil}$ novos casos de TB, com uma incidência de 28,9/100.000 hab. ${ }^{13}$. Durante o período avaliado, na Região Norte (ES) foram notificados 559 casos de TB (2015-2019), sendo: 2015 com 118 casos, 2016 (110 casos), 2017 (104 casos), 2018 ( 112 casos) e 2019 com 115 casos no total, Figura 1.

Dentre todos os casos notificados é possível perceber um maior número de notificações no ano de 2015, sendo São Mateus o município que obteve mais notificações em todos os anos, totalizando $35,06 \%$ de toda Região Norte do estado, seguido de Pedro Canário com $(9,66 \%)$, Nova Venécia $(9,48 \%)$ e Jaguaré (7,87\%), Figura 1. Observa-se menores incidências para os municípios de Água Doce do Norte, Boa esperança, Mucurici e Ponto Belo.

De acordo com a Tabela 1, é possível observar os coeficientes de incidência da doença na região. No período analisado, registraram-se os seguintes coeficientes de incidência para todas as formas de tuberculose na Região Norte do Estado, 27,7/100.000 habitantes (2015); 25,6/100.000 habitantes (2016); 24/100.000 habitantes (2017); 26,3/100.000 habitantes (2018); 26,7/100.000 habitantes (2019).

Quando analisamos os dados de todo país, observa-se que no Brasil, em 2018, foram diagnosticados 75.717 casos no-

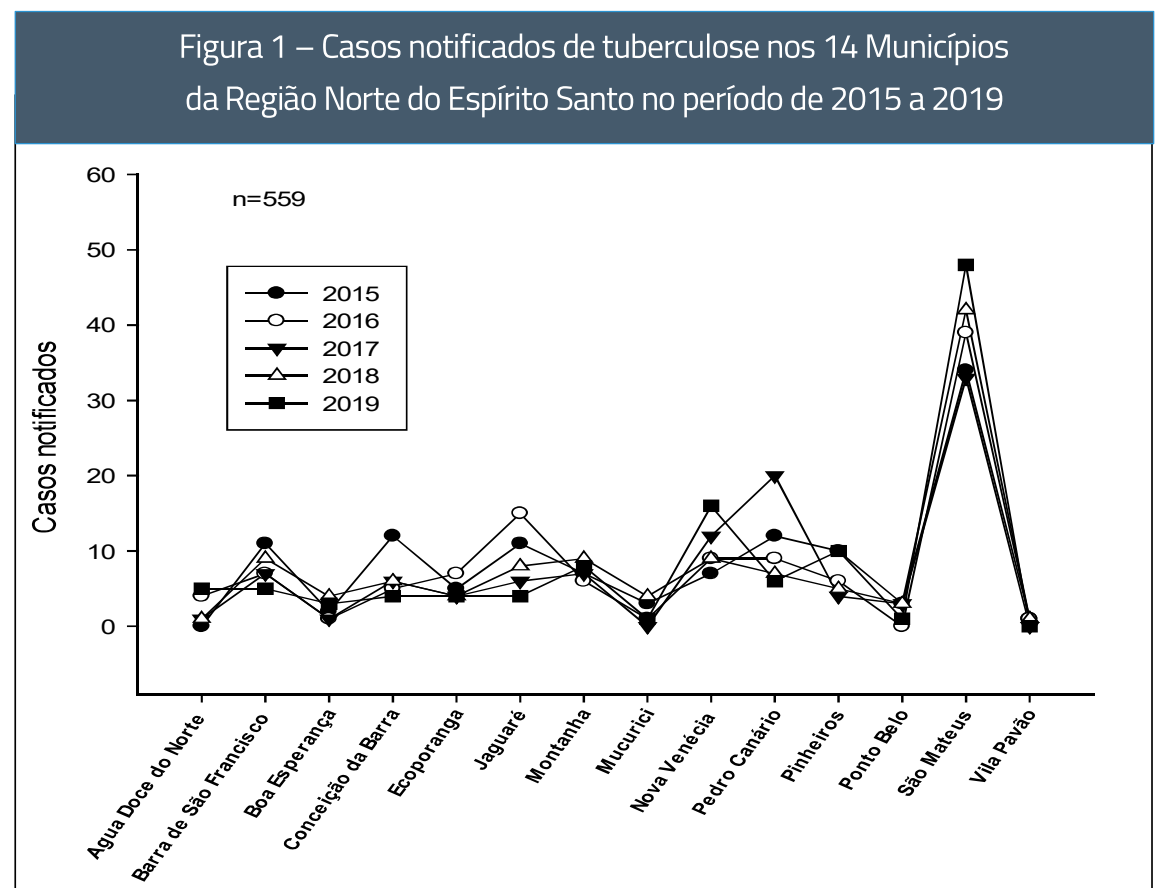

Fonte: SINAN, 2020.

Tabela 1 - Coeficiente de Incidência de Tuberculose nos 14 municípios da região Norte do Espírito Santo no período de 2015 a 2019.

\begin{tabular}{|c|c|c|c|c|c|}
\hline \multirow[t]{2}{*}{ MUNICÍPIOS } & \multicolumn{5}{|c|}{ COEFICIENTE DE INCIDÊNCIA 2015-2019 } \\
\hline & 2015 & 2016 & 2017 & 2018 & 2019 \\
\hline Água Doce do Norte & 0,0 & 33,5 & 8,4 & 9,0 & 45,4 \\
\hline Barra de São Francisco & 24,7 & 15,6 & 15,5 & 20,3 & 11,2 \\
\hline Boa Esperança & 13,1 & 6,5 & 6,5 & 26,7 & 20,0 \\
\hline Conceição da Barra & 38,6 & 15,9 & 19,0 & 19,4 & 12,9 \\
\hline Ecoporanga & 20,6 & 28,9 & 16,5 & 17,4 & 17,4 \\
\hline Jaguaré & 38,4 & 51,5 & 20,2 & 26,8 & 13,1 \\
\hline Montanha & 36,4 & 31,1 & 36,1 & 47,9 & 42,5 \\
\hline Mucurici & 51,0 & 17,0 & 0,0 & 72,0 & 18,1 \\
\hline Nova Venécia & 13,9 & 17,8 & 23,5 & 18,1 & 31,9 \\
\hline Pedro Canário & 45,9 & 34,2 & 75,4 & 26,9 & 22,9 \\
\hline Pinheiros & 37,6 & 22,3 & 14,7 & 18,7 & 37,0 \\
\hline Ponto Belo & 38,7 & 0,0 & 38,0 & 38,5 & 12,7 \\
\hline São Mateus & 27,3 & 30,8 & 25,7 & 32,7 & 36,8 \\
\hline Vila Pavão & 10,7 & 10,6 & 0,0 & 10,9 & 0,0 \\
\hline
\end{tabular}




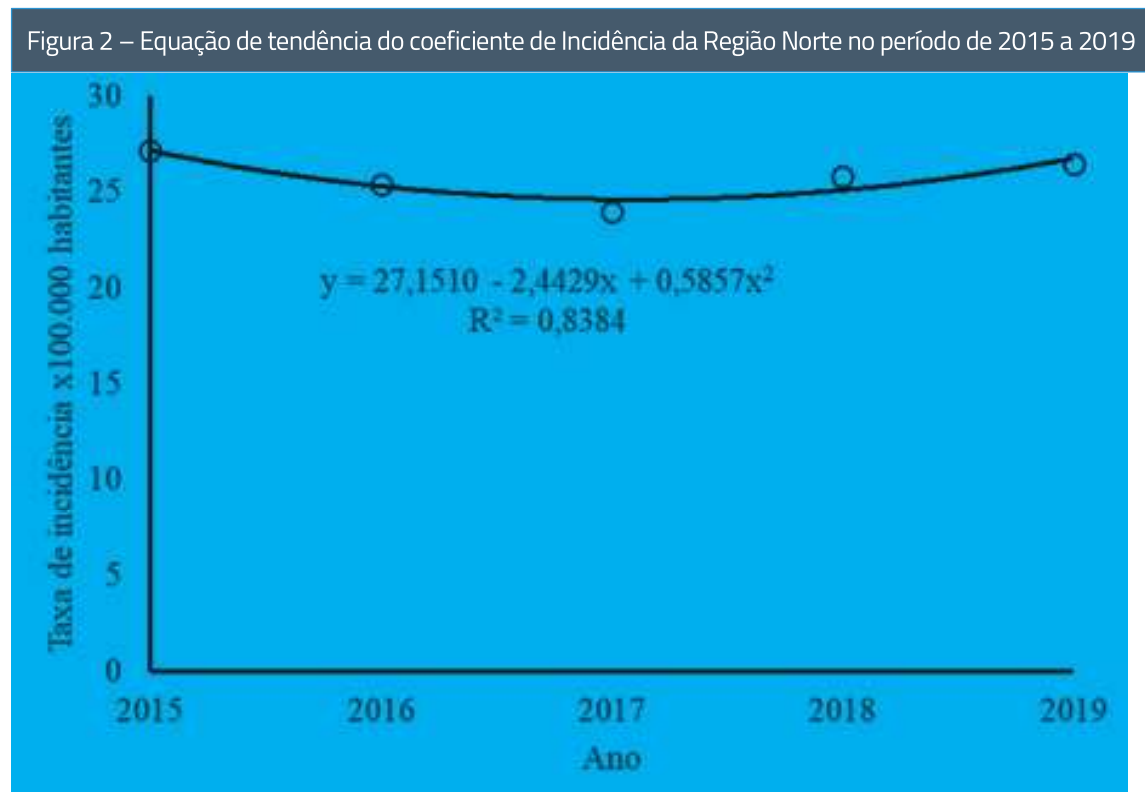

Fonte: Os autores, 2021

vos da doença, obtendo um coeficiente de incidência de 36,2 casos/100 mil hab. Embora, de 2009 a 2018, tenha sido observada uma queda média anual de $1,0 \%$, o coeficiente de incidência aumentou nos anos de (2017) 35,3/100 mil hab. e (2018) $36,2 / 100$ mil hab. sendo que para o ano de (2015) tivemos 34,1/100 mil hab. e (2016) 33,9/100 mil hab. (Brasil, 2019). Fato que na região norte de saúde obtém obteve-se uma diminuição média anual de 2,1\% para o ano de 2015 para 2016, e para o ano de 2016 a 2017 com uma diminuição de 1,6\%, voltando a subir para os anos seguintes, mas com percentuais muito menores. A taxa de incidência entre os anos de 2015 a 2019 foi maior no ano de 2015, onde entre os municípios da região Mucurici teve o maior coeficiente (51). Já em 2016, Jaguaré apresentou um coeficiente de (51,5), em 2017 Pedro Canário com (75,4), 2018 Mucurici (72) e 2019 Água Doce do Norte com $(45,4)$.

$\mathrm{Na}$ figura 2, observa-se a equação de tendência do agravo da região norte do Estado do Espírito Santo nos cinco anos do estudo.

É possível verificar que houve dois comportamentos, um na diminuição para o ano de 2015 a 2017 e voltando a subir de 2018 a 2019. Dessa forma, o coeficiente explica $83,84 \%$ dos fatores que interferem na taxa de incidência para a Região Norte do estado do Espírito Santo. Para o ano de 2015 o coeficiente de incidência em análise foi de (27,2); 2016 (25,4); 2017 (24); 2018 $(25,8)$ e $2019(26,5)$.

\section{DISCUSSÃO}

De acordo com as notificações é possível verificar um maior quantitativo concentrado no município de São Mateus (35,06\%) fato esse que pode ser explicado possivelmente pelo alto índice populacional concentrado no município de São Mateus, totalizando aproximadamente $132.642 \mathrm{mil}$ pessoas de acordo com o censo de 202010.

Quando analisamos o restante dos municípios que compõem a região, observamos um menor número populacional, favorecendo, todavia, um menor número de casos para a doença em comparação a São Mateus. Entretanto, vale salientar que a TB na Região Norte de Saúde do Espírito Santo possui certa estabilidade na ocorrência dessas notificações. De acordo com Pelaquin; Silva e Ribeiro 6, em cidades com urbanização caótica e grandes bolsões de pobreza, as deficiências do sistema de saúde provavelmente justificam o elevado número de casos e óbitos por TB. Quando observamos um menor índice de notificação, à literatura traz alguns fatores que podem estar associados a esses casos, como a má qualidade dos bancos de dados e dos prontuários, que podem vir a trazer dificuldades nas análises desses para avaliação do agravo e consequentemente gerar a perca dessas notificações acarretando na diminuição dos casos registrados incluindo a subnotificação que ocorre inclusive em municípios menores $^{6}$.

Em um estudo de pacientes portadores de TB atendidos em uma unidade municipal de saúde de Belém/PA nos anos de 2011 e 2012, de acordo Freitas; Santos: Silva e Rocha, $2016^{3}$ relata que a doença ainda está sendo registrada frequentemente no sistema básico de saúde, com isso observa-se a necessidade da intensificação de estratégias de prevenção e divulgação das formas de tratamento da doença, objetivando a diminuição da incidência e prevalência de casos a nível local e regional.

É possível destacar esses percentuais que foram identificados na região norte de saúde através de vários fatores, entre eles os altos percentuais de notificação que são de extrema importância para iniciar o tratamento adequado e detectar todos os casos a fim de quebrar o ciclo de transmissão do agravo, como explicado por Junior, 20048, em que postos, centro de saúde, ambulatórios e hospitais - para realização da busca ativa de sintomáticos respiratórios devem ser uma atitude permanente e incorporada à rotina de atividades de todos os membros das equipes de saúde. Esses fatores são de extrema importância, o que leva as instituições a identificar os pacientes, preconizar o tratamento precoce, de do ponto de vista epidemiológico consequentemente a busca ativa com detecção de casos e diagnóstico precoce faz com que haja um aumento no coeficiente de incidência de cada localidade.

Junior, $2004^{8}$ explica ainda, que nas áreas onde as ações já estão organizadas, a visita deve incluir a detecção de casos entre sintomáticos respiratórios e contatos, principalmente de casos bacilíferos e crianças, e quando necessário, deve-se referenciá-los ao serviço de saúde. Quando isso não ocorre, há uma diminuição dos casos e conse- 


\section{artigo}

Sotero, R. L., Gouvea, R. R., Cardoso, M. L., Gomes, A. M., Forza, D. H.,Santos, G. L.

Aspectos epidemiológicos da tuberculose na região norte de saúde do estado do Espírito Santo (2015 - 2019)

quentemente menor incidência do agravo na região, deixando com que os casos circulem sem receber o tratamento adequado e propagando ainda mais os bacilos de Koch inclusive em ambientes domiciliares. Sabe-se que a busca ativa dos casos de TB ainda não se enquadra em uma ação prioritária nos serviços de saúde, embora o paciente ter uma ampliação do acesso as ferramentas de diagnóstico é uma estratégia útil no controle da doença1;15. E quando verificamos uma diminuição entre os anos de 2015 a 2017, pode-se explicado possivelmente em decorrência da implantação de políticas públicas de controle do agravo ${ }^{11}$.

$\mathrm{Na}$ distribuição dos coeficientes de incidência na equação de tendência, Peternelli, 20129 explica que a análise consiste na realização de uma estatística com o objetivo de verificar a existência de uma relação funcional entre uma variável dependente com uma ou mais variáveis independentes, fato esse que é explicado pelos dados já apresentados, onde foi observado um coeficiente maior para os anos de 2015 e 2019 e consequente decrescente no período de 2015 a 2017, voltando a subir em 2018. E com isso, sugere-se algumas ponderaçōes no momento de se escolher o modelo, entre eles deve ser condizente tanto no grau como no aspecto da curva, para representar o fenômeno em estudo ${ }^{9}$.

\section{CONCLUSÃO}

Os achados demonstram que a região tem demonstrado resultados relevantes sob a ótica do panorama nacional e estadual em relação ao combate e enfretamento da patologia.

O que demonstra que diante de inovaçōes terapêuticas e profiláticas, ainda que não acompanhando o agravo sob vigilância constante conhecendo fatores de incidên- cia e distribuição dos casos mediante a um bom diagnóstico territorial exemplifica esforços combinados dos diversos setores da sociedade para implementação de medidas mitigadoras e alcance de uma saúde que seja integral, igualitária e equânime como sugerido pelos princípios norteadores do sistema único de saúde. Ressalta-se que há a necessidade da realização de mais pesquisas acerca do tema a fim de conhecer a realidade da tuberculose em determinada região, caracterização dos portadores bem como os possíveis riscos a fim de evitar a disseminação da doença e o aumento da mortalidade.

Cabe ainda ratificar, que um serviço de vigilância bem estruturado em determinada região de saúde corrobora para que o acesso à saúde dos usuários se dê de forma não sumária e colaborando para que o indivíduo seja protagonista do seu cuidado.

\section{REFERÊNCIAS}

1. Cecílio HPM, Higarashi IH, Marcon SS. Opinião dos profissionais de saúde sobre os serviços de controle da tuberculose. Acta paul. Enferm. 2015; 28(1): 19-25

2. Silva, DR., Mello, FCDQ., Migliori, GB. Tuberculosis series 2020. Jornal Brasileiro de Pneumologia, n. 46, v.2, 2020

3. Freitas, WMTM.; Santos, CC.; Silva, MM.; Rocha, GA. Perfil clínico-epidemiológico de pacientes portadores de tuberculose atendidos em uma unidade municipal de saúde de Belém, Estado do Pará, Brasil. Revista Pan-Amaz Saúde, 2016, v.7, n.2, p. 45-50

4. Secretaria de Estado da Saúde do Espírito Santo (SESA - ES). Disponível em:<https://saude.es.gov.br/programa-estadual-de-controle-da-tuberculose>. Acesso em 16 de agosto de 2020

5. Brasil. Ministério da Saúde. Departamento de informática do SUS- DATASUS. Informações de saúde, epidemiológicas e morbidade: banco de dados. 2012. Disponivel em: http://www2.datasus.gov.br/DATASUS/index.php?area $=0203$.

6. Pelaquin, MHH.; Silva, RS.; Ribeiro, SA. Fatores associados ao óbito por tuberculose na zona leste da cidade de São Paulo, 2001. Jornal Brasileiro de Pneumologia, UNIFESP - São Paulo (SP) Brasil, 2007; n (33), v (3), p. 311317.

7. Freitas, WMTM.; Santos, CC.; Silva, MM.; Rocha, GA. Perfil clínico-epidemiológico de pacientes portadores de tuberculose atendidos em uma unidade municipal de saúde de Belém, Estado do Pará, Brasil. Revista Pan-Amaz Saúde, 2016, v.7, n.2, p. 45-50.

8. Junior, JB Da Silva. Tuberculose Guia de Vigilância Epidemiológica. Jornal Brasileiro de Pneumologia, 2004, n30, p. 57-85.
9. Peternelli, L. A. Capítulo 2: estatística descritiva. Material didático. 2012. Disponivel em:< http://www. dpi. ufv. br/ peternelli/inf162. www, 16032004. Acesso em 16 de novembro de 2020

10. IBGE, Instituto Brasileiro de Geografia e Estatística. Panorama do Município de São Mateus- ES. Disponivel em <https://cidades.ibge.gov.br/brasil/es/sao-mateus/panorama>. Acesso em 14 de agosto de 2020.

11. Marques L.C.S.; Oliveira, O.L.S.; Pereira, M.A.;Perfil clínico, epidemiológico e laboratorial da tuberculose entre 2014 a 2019 no estado da Bahia. Revista Saude Coletiva. 2020; (10) N.57: 3523- 3528.

12. Ministério da Saúde (BRASIL). Secretaria de Vigilância em Saúde. Vigilância em saúde no Brasil 2003 A 2019: da criação da Secretaria de Vigilância em Saúde aos dias atuais. Boletim Epidemiológico. 2019 set, 50(n.esp.):1154. Disponivel em: http://www.saude.gov.br/boletins-epidemiologicos.

13. Ministério da Saúde (BRASIL). Secretaria de Vigilância em Saúde.Boletim Epidemiológico de Tuberculose. Número Especial | Mar. 2021: 11-43. Disponivel: https:/www.gov.br/saude/pt-br/media/pdf/2021/marco/24/ boletim-tuberculose-2021_24.03\#: :text=Em\%202019\%2C\%20foram\%20 notificados\%20cerca,\%C3\%B3bitos\%20por\%20100\%20mil\%20habitantes.

14. World Health Organization. Global Tuberculosis Report. [Inter-net]. Geneva. 2020. Disponivel em:https://apps.who.int/iris/bitstream/handle/106 65/336069/9789240013131-eng.pdf. Acesso: 06 de agosto de 2021.

15. Souza, G.A.S.C.; Ferreira, M.R.L.; Bonfim, R.O.; Órfão, N.H.;Perfil de saúde da tuberculose entre crianças e adolescentes indígenas: uma revisão integrativa. 2021; (11) N.65: 5570-5979Marques L.C.S.; Oliveira, O.L.S.; Pereira, M.A.;Perfil clínico, epidemiológico e laboratorial da tuberculose entre 2014 a 2019 no estado da Bahia. Revista Saude Coletiva. 2020; (10) N.57: 35233528. 